\title{
Pâturage et cultures techniques de l'herbe
}

Les mondes de l'élevage des territoires de montagne du Vercors et de la Bigorre

Grazing and technical grass cultures. The world of livestock breeding in the mountain regions of the Vercors and the Bigorre

Antoine Doré, Claude Compagnone, Laurent Dobremez et Sophie Madelrieux

\section{(2) OpenEdition}

\section{Journals}

Édition électronique

URL : https://journals.openedition.org/tc/7386

DOI : $10.4000 /$ tc. 7386

ISBN : 0248-6016

ISSN : 1952-420X

Éditeur

Éditions de l'EHESS

\section{Édition imprimée}

Date de publication : 1 mai 2015

Pagination : 56-73

ISBN : 9782713224874

ISSN : 0248-6016

\section{Référence électronique}

Antoine Doré, Claude Compagnone, Laurent Dobremez et Sophie Madelrieux, « Pâturage et cultures techniques de l'herbe », Techniques \& Culture [En ligne], 63 | 2015, mis en ligne le 01 mai 2018, consulté le 29 septembre 2022. URL : http://journals.openedition.org/tc/7386 ; DOI : https://doi.org/10.4000/ tc.7386 


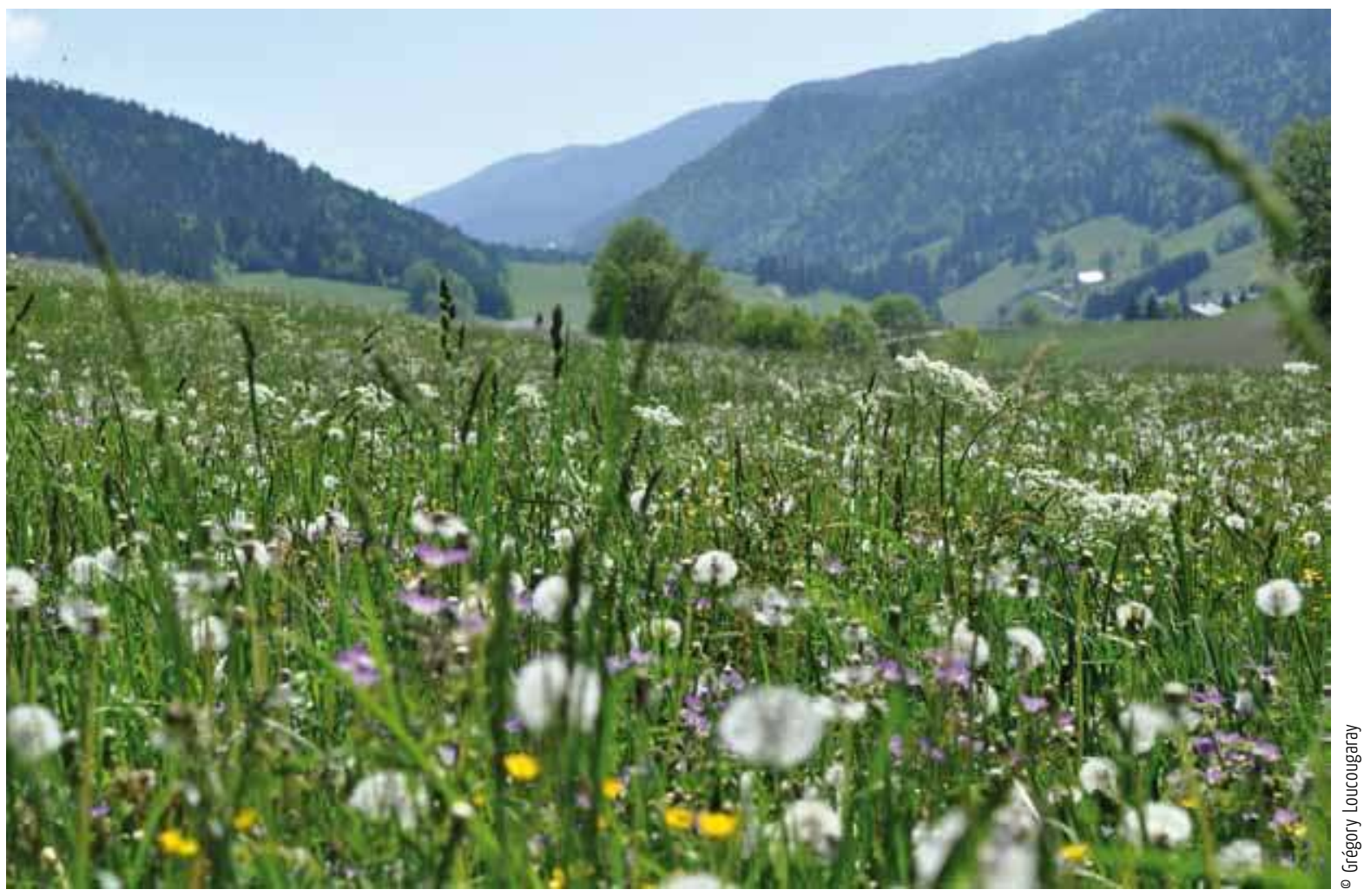




\section{PÂTURAGE ET CULTURES TECHNIQUES DE L'HERBE Les mondes de l'élevage des territoires de montagne du Vercors et de la Bigorre}

Au cours des dernières décennies, le triptyque « famille - progrès - régulation étatique » qui a structuré le modèle agricole républicain français pendant les Trente Glorieuses (Barthez, 1982; Lémery, 2003; Rémy, 1987) s'est progressivement fragilisé sous l'effet d'une série de changements aussi bien techniques, économiques et environnementaux que sociaux et politiques. L'effacement relatif d'un secteur agricole économiquement régulé (Fouilleux, 2008; Muller, 2010), corrélatif d'une inscription plus marquée de l'agriculture dans un marché concurrentiel (Hervieu \& Purseigle, 2013), amène les agriculteurs à tenir en tension deux exigences. L'une porte sur leur insertion dans un mouvement de globalisation, qui met en jeu leur capacité à s'inscrire dans des marchés internationaux incertains; l'autre concerne leur positionnement dans un mouvement de (re)territorialisation des productions, qui met en jeu leur capacité à s'insérer dans un tissu d'activités hétérogènes et à établir des partenariats élargis avec toute une série d'acteurs nouveaux qui n'intervenaient pas antérieurement dans l'orientation de l'agriculture (Mormont, 2009).

Dans une telle situation, la gestion des ressources dites « naturelles » devient un point sensible de l'articulation, dans un régime de proximité ou de distance, des lieux de production et des lieux de consommation des produits de l'alimentation humaine, mais également des lieux de production et des lieux d'accès aux ressources nécessaires à la production telles que les fourrages et les céréales dans le cas de l'élevage herbivore.

L'objectif de cet article est de décrire et d'analyser la façon dont les activités d'élevage herbivore des territoires de montagne s'appuient sur l'usage des ressources herbagères locales. En nous focalisant sur l'élevage de montagne, nous voulons faire apparaître

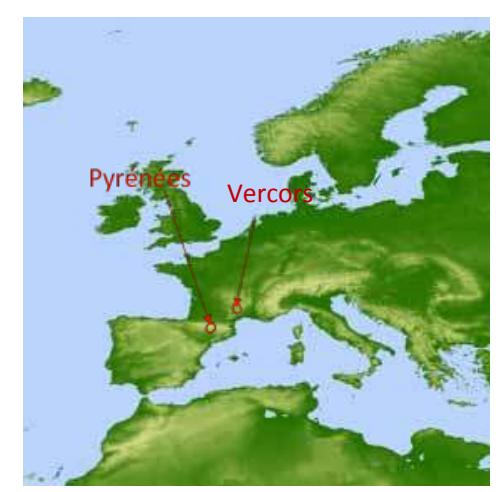




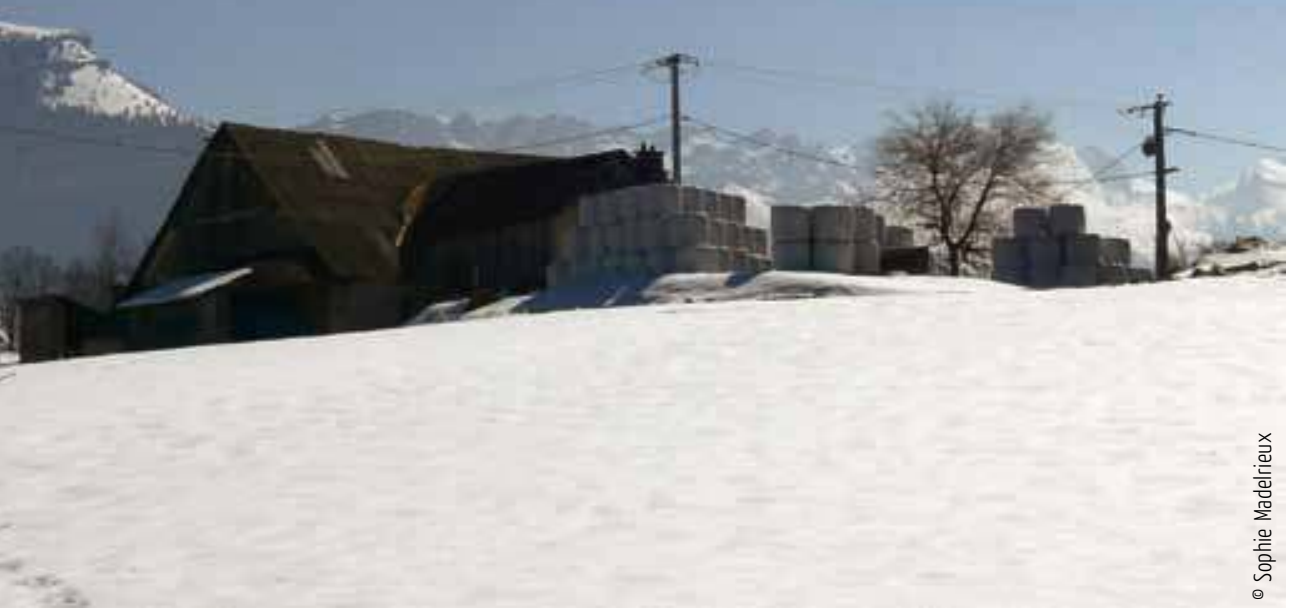

Les éleveurs doivent faire face à une durée d'hivernage de l'ordre de 5-6 mois durant laquelle les animaux restent enfermés dans les bâtiments. Ici une grange dans les Quatre

Montagnes avec le stockage du foin enrubanné à l'extérieur. la diversité des modalités d'articulation entre les lieux de production et les lieux de disponibilité des ressources dans des régions soumises à des contraintes de milieux importantes, variables dans le temps (contrastes saisonniers) et dans l'espace (contrastes pédoclimatiques). Pour ce faire, nous rendons compte, dans une démarche comparative, des cultures techniques de l'herbe d'éleveurs du Vercors (Alpes du Nord) et d'éleveurs de la Bigorre (Pyrénées). Cette démarche comparative permet de se donner les moyens d'interroger à la fois « l'allant de soi » et « l'absent » sur chaque terrain: « l'allant de soi », qui n'est pas discuté sur un terrain, se trouve questionné quand il n'est précisément pas le même d'un terrain à un autre; « l'absent » sur un terrain se révèle en creux lorsque des éléments sont présents sur un terrain et pas un autre. Dans une approche compréhensive des pratiques cet article prend appui sur les données tirées d'entretiens menés auprès de 55 éleveurs (22 dans le Val d'Azun et 33 dans le Vercors) dans le cadre d'une étude portant sur la redéfinition des pratiques et des identités professionnelles dans les mondes de l'élevage (Doré, 2013). L'analyse tire également parti de deux enquêtes conduites auprès de différents acteurs (éleveurs, élus locaux, représentants d'associations environnementales, professionnels du tourisme, etc.) du Vercors et de la Bigorre sur le rôle qu'ils attribuent à l'élevage sur leur territoire (Barnaud \& al., (accepté); Dobremez \& al., 2012), ainsi que d'une analyse des systèmes fourragers des éleveurs du Val d'Autrans dans les Quatre Montagnes (Dobremez \& al, 2013) ${ }^{1}$.

Sur ces deux territoires, les éleveurs doivent faire face à une durée d'hivernage de l'ordre de 5 à 6 mois durant laquelle les animaux restent enfermés dans les bâtiments et où le recours au pâturage est impossible. Les solutions alternatives (transhumance hivernale ou mise en pension des animaux en plaine) sont rarement utilisées et ne concernent alors souvent qu'une partie du troupeau (génisses). L'alimentation des troupeaux pour la production de lait ou de viande s'appuie alors sur l'utilisation de fourrages conservés (foin et ensilage qui forment la ration de base) et d'aliments concentrés (céréales, tourteaux, etc., pour complémenter la ration). La viabilité économique des exploitations dépend souvent de la capacité des éleveurs à réduire les charges liées à l'achat d'aliments, notamment de fourrages. Les conditions d'exercice des activités d'élevage dans ces territoires de montagne sont alors étroitement liées aux modalités sociotechniques d'usage et de valorisation des ressources herbagères locales (Doré, 2013).

Ces deux terrains d'étude se différencient notamment par la place que le pâturage y occupe. D'un côté, en Val d'Azun dans la Bigorre (Canton d'Aucun dans les 
Disposer de surfaces fourragères suffisantes est un enjeu particulièrement important pour la grande majorité des éleveurs du Val d'Azun. D’une part, les marges de manœuvre pour l'intensification de la production d'herbe sont très faibles car la mécanisation des parcelles est limitée par des contraintes topographiques importantes. D’autre part, l'accès aux pâturages est borné dans le temps par des contraintes climatiques fortes. On bascule dans le Val d'Azun d'un élevage quasiment hors-sol en hiver à un élevage extensif en été, en passant par un élevage relativement intensif à la mi-saison. À cette période les animaux restent généralement concentrés sur les prairies où la neige s'est retirée. La pression exercée sur les pâturages est donc importante. Dans de telles conditions, la sécurisation des systèmes de production passe par une gestion différenciée des espaces, qui intègre des contraintes naturelles et sociales d'accès aux espaces fourragers.

Les éleveurs ont tout d'abord recours de manière généralisée aux estives. « La montagne » est généralement considérée comme une ressource nécessaire sans laquelle aucune activité professionnelle d'élevage ne pourrait être maintenue sur le canton: «Le fait de transhumer nous double la surface agricole ». Même si l'usage des estives est valorisé sur le plan qualitatif et sert d'argument à la promotion et à la commercialisation du fromage et de la viande, les éleveurs l'envisagent davantage sur le plan quantitatif lorsqu'ils sont amenés à décrire leur système de production. La mise en estive des animaux permet notamment de récolter plus de foin pour l'hiver sur les prairies libérées des zones intermédiaires. Il s'agit alors d'articuler production de foin et pâturage dans l'organisation du système de production. Comme en témoigne cet éleveur de vaches et de brebis laitières:

« Les terrains que j'ai, à partir du mois de mai, il ne faut plus de bétail dessus. J'ai besoin du fourrage pour passer tout l'hiver ».

L'usage de ces ressources herbagères d'altitude suppose un certain nombre de compromis zootechniques et d'arbitrages collectifs parfois conflictuels. Premièrement, sur le plan zootechnique, tous les animaux ou tous les types de production ne sont pas adaptés aux conditions de vie en estive. Il s'agit de privilégier les animaux résistants, au détriment parfois de certaines races plus productives mais trop fragiles pour la montagne. Pour cet éleveur ovins-viande par exemple: « Le problème, c'est la rusticité [des animaux]. $\mathrm{Vu}$ l'utilisation qu'on a des estives... on ne peut pas passer à côté ». Dans le cadre des systèmes laitiers, l'usage des ressources herbagères d'altitude est également limité par l'obligation de cantonner les animaux à proximité des routes et des

\section{Zone Pastorale}

Les éleveurs du Val d'Azun ont recours de manière généralisée aux estives. Une signalétique pastorale a été conçue afin d'informer les touristes

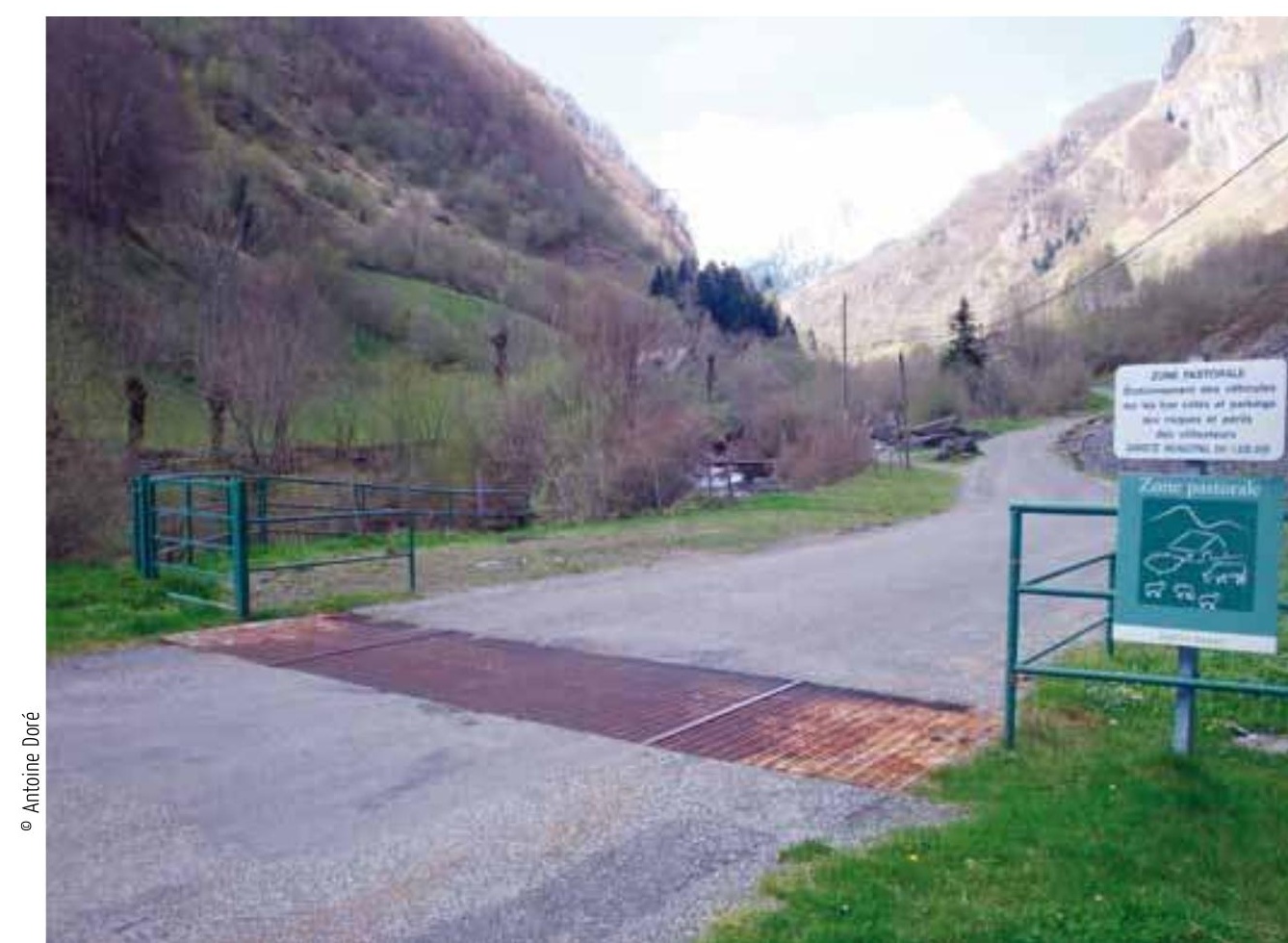


pistes carrossables que l'éleveur peut emprunter quotidiennement pour venir faire la traite et pour ramener le lait à la ferme ${ }^{5}$. Deuxièmement, tous les éleveurs n'ont pas accès de manière équivalente à ces ressources herbagères pour des raisons qui tiennent à la façon dont cet accès est socialement organisé et contrôlé par certains éleveurs (Compagnone, 2008; Eychenne, 2008). Les espaces d'altitude relèvent généralement de la propriété collective communale (ou intercommunale) et les éleveurs dont le siège d'exploitation est situé sur la commune sont considérés comme des ayants droit. Toutefois, les estives sont généralement partagées en différents secteurs et les éleveurs ne se voient pas attribuer des secteurs de la même qualité. Ainsi certains de ces secteurs sont situés à proximité d'une piste ("Ils te posent la bagnole et bon... ils marchent un peu, mais ça va! ») alors que d'autres sont plus difficiles d'accès ("Nous, là où on est, il y a déjà quatre heures de marche pour y monter »). Les conditions d'accès aux bonnes estives mettent en jeux des relations de pouvoir relativement complexes qui reposent, entre autres, sur l'ancienneté intergénérationnelle d'exploitation familiale de ces espaces (les nouveaux arrivants se retrouvent généralement relégués dans les zones difficiles) et sur la proximité aux fonctions professionnelles et politiques locales.

L'exploitation des zones intermédiaires reste difficile : les labours sont souvent impossibles; la configuration des parcelles oblige les éleveurs à faucher lentement (pour éviter de casser le matériel du fait de la présence de rochers ou pour ne pas détériorer la qualité du foin au moment de la coupe en passant, en raison des irrégularités de la surface des parcelles, la lame de fauche dans la terre); les dates de fauche ne peuvent être planifiées avec précision en raison des contraintes météorologiques et de cette configuration particulière des parcelles. Quelques éleveurs tentent d'améliorer les choses en aménageant, par exemple, certaines portions de parcelles: «Il y avait des plateaux où on a fait arracher les cailloux pour pouvoir passer le matériel $»$. La question du mode d'exploitation de ces parcelles n'apparaît vraiment dans les débats entre éleveurs qu'à travers les critiques que certains adressent aux autres sur l'abandon des anciennes pratiques d'entretien pour des raisons d'économie de travail. Ils leur reprochent de « ne pas assez s'occuper » des prairies et donc de « gaspiller l'herbe », de ne pas «nettoyer » ou «faire bien propre ${ }^{6}$, en ne faisant pas « les bordures », en ne ratissant pas les résidus de foin laissés derrière le passage des presses à bottes, en abandonnant la fauche pédestre des «mauvaises terres » non mécanisables, etc.

Au-delà des questions relatives aux modalités d'exploitation des parcelles de ces zones intermédiaires, ce sont surtout les problèmes liés à leur accessibilité qui sont au coeur des discussions et des préoccupations des éleveurs sur Val d'Azun. En effet, de nombreuses granges foraines ${ }^{7}$ se trouvent dispersées dans cet espace. Les éleveurs y répartissaient traditionnellement leur troupeau pendant l'hiver et l'intersaison. Beaucoup de ces granges ont été vendues pour être aménagées en habitations. Les éleveurs cédant avec ces granges toute ou partie de la surface des parcelles sur lesquelles elles sont installées, l'accès aux ressources herbagères est alors subordonné au bon vouloir de ces nouveaux propriétaires. Ces derniers refusent généralement d'établir des baux de location. Ils peuvent ainsi décider à tout moment de se séparer d'un éleveur et de proposer leur parcelle à un autre s'ils estiment, selon les éleveurs enquêtés, que celui-ci ne travaille pas « proprement », «n'entretient pas assez bien» les parcelles, « amène des mouches avec ses bêtes », etc. Lorsque le propriétaire est issu d'une famille d'éleveurs, la pression exercée sur l'éleveur peut être encore plus importante, comme l'explique cet éleveur de brebis tarasconnaises: 


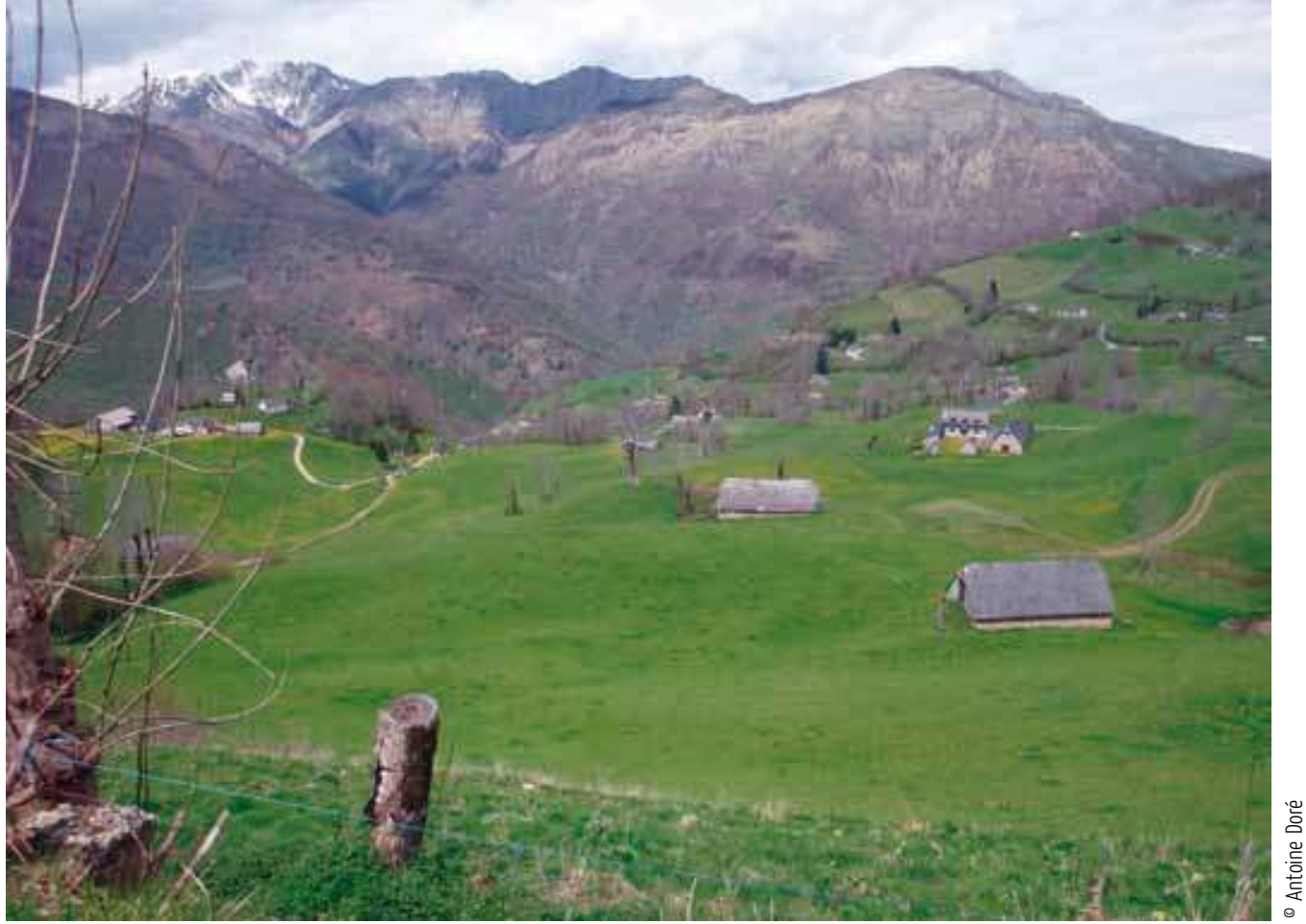

« Les pires, des fois, ce sont ceux qui sont issus du milieu agricole de la génération de l'avant qui reviennent et qui ne comprennent pas que les terrains ne sont pas aussi bien entretenus qu'avant. Parce qu'ils ont connu leurs pères qui ratissaient chaque coin de pré ».

La sécurisation de l'accès aux ressources fourragères passe enfin, pour certaines des plus grosses exploitations, par l'acquisition ou la location de parcelles en plaine. Des éleveurs n'hésitent pas ainsi à faire une cinquantaine de kilomètres avec leur matériel agricole pour aller cultiver quelques hectares de maïs ou récolter du foin. Le temps et l'énergie dépensés dans les trajets s'avèrent rapidement rentabilisés:

«Là-bas on va faire 100 boules [de foin] avec deux doigts dans le nez; que de faire 20 boules ici, on aura cassé la moitié d'une rotative contre un caillou. On aura passé trois jours à faire 20 boules de foin ».

À travers cette petite expérience du travail de l'herbe en plaine, les éleveurs découvrent des conditions de travail très différentes de celles dont ils ont l'habitude en montagne.
Dans Val d'Azun, la sécurisation de l'accès aux ressources fourragères passe par l'exploitation des zones intermédiaires pontées de granges foraines: « [La grange] ça peut être un capital économique [nous explique un éleveur de brebis et de vaches laitières]; pour un jeune, s'il en vend une, il peut construire un hangar et il peut s'installer sans emprunt. Ça résout les problèmes de partage de famille, et ça peut être un revenu complémentaire si on l'emménage en gite. [...]. Et les communes ne peuvent pas mettre ces zones en zones agricoles pour ça ».

\section{Exploiter la prairie en Vercors}

À proximité de Grenoble, le territoire des Quatre Montagnes est tourné vers l'élevage et le tourisme. Les fermes coexistent avec des stations de ski nordique et de ski alpin. L'élevage de bovins et la production de fromages sont des activités traditionnelles de cette partie du Vercors qui bénéficie notamment de l'Appellation d'Origine Protégée (AOP) « Bleu du Vercors-Sassenage ». L'essentiel des volumes de lait produit est collecté et transformé par la coopérative Vercors-Lait mais un quart des exploitations de bovinslait transforme et commercialise une partie du lait à la ferme. Ici - comme sur l'ensemble 


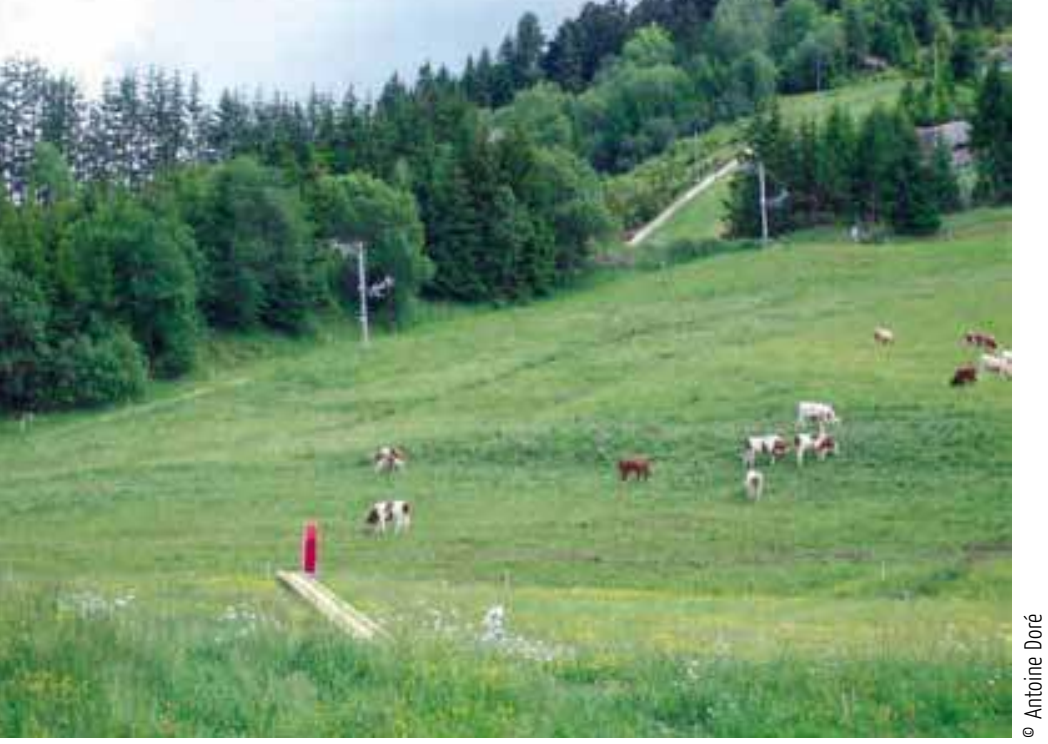

Dans les Quatre Montagnes, les pistes de ski sont pâturées. du territoire national - le nombre d'exploitations agricoles a été divisé par deux au cours des trente dernières années, tandis que la taille moyenne des troupeaux de vaches laitières a plus que doublé ${ }^{8}$. Les exploitations sont principalement des exploitations familiales mais les ménages ayant une activité exclusivement agricole sont désormais minoritaires ${ }^{9}$. Le conjoint travaille souvent à l'extérieur de l'exploitation et beaucoup d'éleveurs ont une activité saisonnière liée notamment aux stations de ski (cours de ski, fonctionnement des remontées mécaniques, damage des pistes, etc.), plus occasionnellement à l'exploitation forestière.

Le paysage agricole des Quatre Montagnes est un paysage de prairies. Le recours aux estives reste confidentiel et ne concerne que quelques génisses. Les surfaces fourragères sont largement prépondérantes ${ }^{10}$, reléguant les cultures de céréales à la portion congrue. Ici, beaucoup préfèrent «cultiver l'herbe» :

«J'ai arrêté de faire de la céréale. Avant, je faisais un hectare d'orge par an. Et maintenant, je laboure, et je sème de l'herbe tout de suite».

L'intensification de la production fourragère est un enjeu particulièrement important pour la grande majorité des éleveurs des Quatre Montagnes. D’une part, l'agriculture est confrontée à une pression foncière due à l'urbanisation et à une recrudescence des aléas (notamment plusieurs épisodes de sécheresse depuis 2003 et, plus récemment, des pullulations de campagnols). D'autre part, l'AOP « Bleu du Vercors-Sassenage » interdit l'achat de fourrages hors zone. En outre, près d'un quart des éleveurs est en agriculture biologique et doit faire face à un coût très élevé des aliments certifiés Bio. Il s'agit alors de sécuriser les systèmes fourragers et d'atteindre l'autonomie fourragère en mettant en place une conduite différenciée des prairies.

L'intensification de la production fourragère se traduit premièrement par un recours accru au labour pour l'installation de prairies temporaires ${ }^{11}$ qui permettent de faire plusieurs coupes annuelles.

« On sait que quand on laboure, on a des prairies dont le rendement est plus important ». « C'est sûr, il faut labourer, il faut ensemencer. Parce que, quand tu fais trois coupes au lieu d'en faire qu'une, c'est quand même pas la même musique ».

L'origine de ce type de mouvement d'intensification de la production herbagère est, pour les éleveurs, généralement économique:

« On a recommencé à labourer en 2005 ou 2006 [...]. Au moment de l’augmentation [du prix] des céréales ».

Toutefois la surface en prairie permanente reste majoritaire. En effet, d'une part, certains éleveurs font le choix de ne pas labourer considérant, comme ce chef d'une des plus grandes exploitations laitières des Quatre Montagnes, que les gains de production ne compensent pas les surcoûts liés à l'usage de matériels, au fioul consommé, à l'achat des semences, au travail fourni, etc.: 
« Je trouve, par rapport à des GAEC qui labourent beaucoup, des dizaines d'hectares par an, c'est sûr qu'ils font peut-être beaucoup plus d'herbe mais si on ramène le coût de mise en œuvre et par rapport à derrière ce qu'il ressort, je ne suis pas convaincu... ».

D'autre part, tous les éleveurs doivent composer avec une part de terres difficilement mécanisables, notamment en raison de fortes pentes. Ils mettent alors en place une conduite différenciée des prairies dans laquelle la pratique du pâturage peut jouer un rôle important dans la valorisation des prairies permanentes. La pratique de la fauche est souvent combinée sur une même parcelle à celle du pâturage.

L'intensification de la production fourragère se traduit deuxièmement par une spécialisation de l'usage des surfaces. Ce type d'intensification est généralement mis en œuvre par des producteurs laitiers. Ils réservent les prairies temporaires à la fauche et mettent en place, sur les prairies permanentes, des pratiques de pâturage relativement intensives sur lesquelles nous reviendrons plus loin. Pour autant, qu'il s'agisse de ne pas travailler les terres à proximité du domicile afin de dissocier la vie familiale et la vie de l'exploitation ou de réserver des parcelles plates pour le pâturage à proximité du siège d'exploitation afin de laisser les animaux à proximité de la salle de traite le soir et de leur éviter durant la journée deux trajets vers les pâtures éloignées, toutes les parcelles labourables ne sont pas travaillées pour y installer des prairies temporaires.

L'intensification de la production fourragère passe aussi par un travail de rationalisation des dates de fauche. Dans le Vercors, cette démarche est, bien entendu, contrainte par les conditions climatiques: «chez nous, on est tenu quand même par le climat ». Mais dans un contexte où la configuration topologique des parcelles permet une mécanisation généralisée du travail, la récolte de ressources herbagères est relativement rapide. Les éleveurs ont donc un nombre de plages d'intervention plus important que dans des conditions plus contraignantes. Toutefois choisir la date optimale de fauche revient à chercher la quadrature du cercle. Il s'agit en effet de trouver le bon compromis entre quantité et qualité des fourrages récoltés. De ce point de vue, les pratiques des éleveurs laitiers et des éleveurs de vaches à viande diffèrent assez nettement. Les premiers cherchent à faucher tôt pour récolter une herbe riche en azote tandis que les seconds laissent pousser l'herbe afin de récolter de plus grands volumes d'un fourrage plus riche en fibre et plus digeste pour les animaux. Mais les pratiques de fauche diffèrent aussi au sein des éleveurs laitiers. Chez ces derniers, la date de fauche constitue un critère de distinction important pour caractériser et situer la pratique des uns et des autres dans le collectif. D’un côté, il y a les partisans de la «fauche précoce » comme cet éleveur de vaches montbéliardes: " [Je fauche] le plus tôt possible. Pour avoir une super qualité ». D’un autre, il y a les partisans de la « fauche tardive » qui cherchent à récolter des volumes suffisants de fourrage comme cet autre éleveur laitier: "Je ne suis pas un partisan de
Dans un contexte où la configuration des parcelles du plateau du Vercors permet une mécanisation généralisée du travail, la récolte de ressources herbagères est relativement rapide et les éleveurs peuvent mieux maîtriser les dates de fauche. 


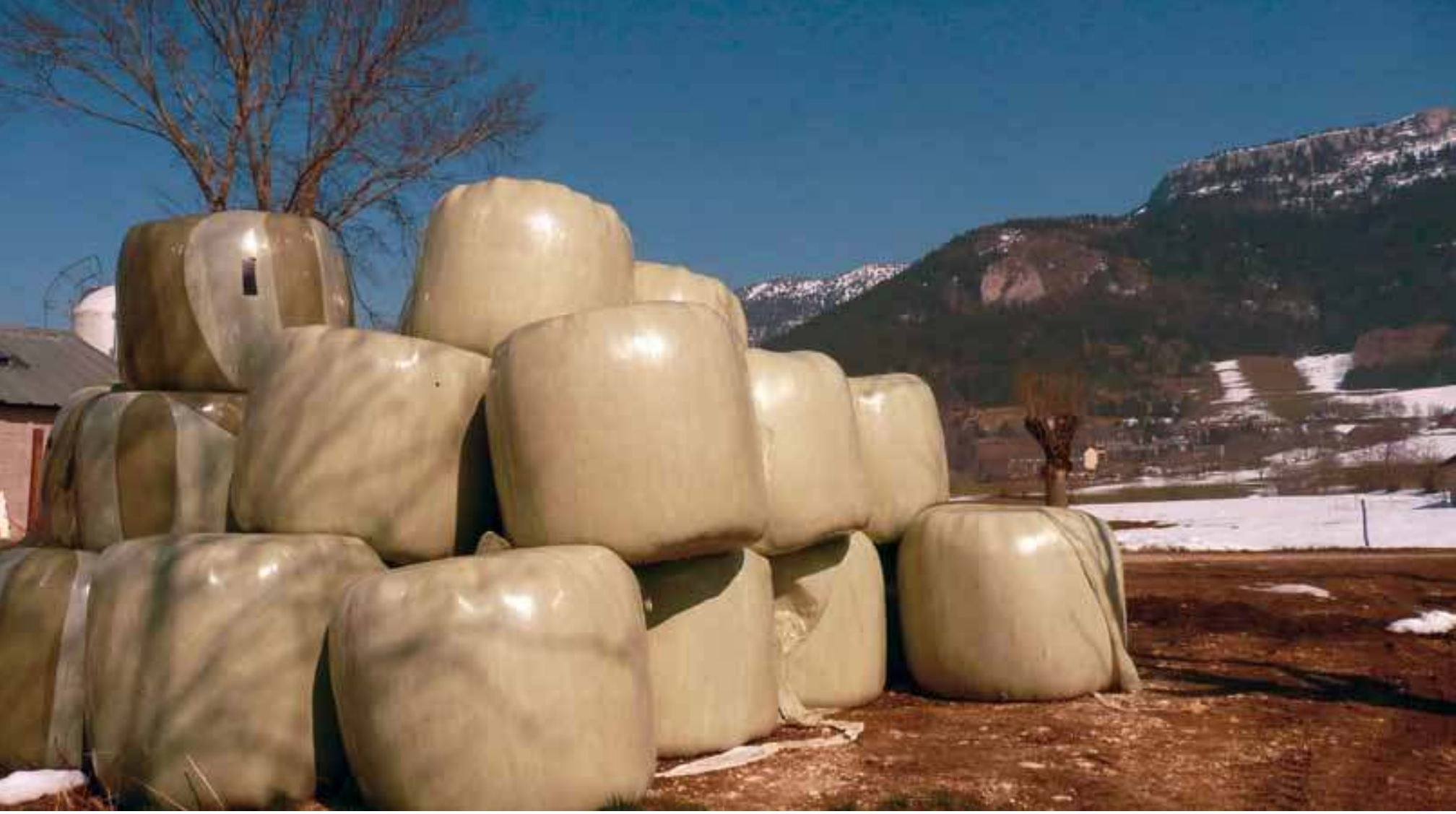

Une fois enrubannés,

QSophie Madelrieux

les fourrages présentent

l'avantage de pouvoir être

stockés à l'extérieur

et de faire l'économie

d'un bâtiment.

faucher trop tôt parce que bon, il faut quand même laisser un peu pousser le foin, par souci d'autonomie ». Ces préférences particulières concernant la date de fauche sont parfois intimement liées à la spécificité des pratiques de pâturage en place. Un éleveur laitier indique ainsi qu’ayant délaissé le «pâturage au fil » (pâturage rationné en déplaçant régulièrement un fil de clôture électrique délimitant la surface d'herbe nécessaire à la ration quotidienne du troupeau) au profit d'un système en « gazon court » (consistant à faire pâturer le troupeau sur un seul parc, en maintenant constamment une hauteur d'herbe limitée à une dizaine de centimètres ${ }^{12}$ ) il se voit dans l'obligation de fournir à ses animaux un foin riche en fibre et donc de pratiquer un peu de fauche tardive:

« Le problème de l'herbe, du gazon court, c'est qu'elles ont toujours de l'herbe rase. [...] ça veut dire qu'il y a trop d'azote dans la ration. [Les vaches] ont des problèmes de fertilité et des problèmes de pied. [Alors] je leur donne du foin à volonté. Elles ont du foin toute l'année. [...] Elles ont du vieux foin. Du foin tardif ».

Lintensification de la production fourragère se traduit enfin dans les pratiques de conservation des ressources herbagères. En prévision de la période d'hivernage, il est important de constituer des stocks fourragers suffisants sur le plan quantitatif d'une part - pour passer les 5-6 mois durant lesquels les animaux restent dans les bâtiments -, et sur le plan qualitatif d'autre part - pour limiter l'achat d'aliments concentrés, notamment chez les éleveurs laitiers. L'ensilage d'herbe ou de maïs étant interdit par le cahier des charges de l'AOP « Bleu du Vercors-Sassenage », les éleveurs ont souvent recours à l'enrubannage - moins fréquemment au séchage en grange - pour constituer un stock fourrager en complément de celui constitué par le foin séché au sol. Cette technique de l'enrubannage, qui consiste à conditionner l'herbe récoltée sous forme de balles sous 
un film plastique hermétique, s'est très largement répandue dans les Quatre Montagnes au cours des trente dernières années. Elle permet de stocker les balles à l'extérieur et de faire ainsi l'économie d'un bâtiment :

« Le coût d'un bâtiment avec le coût d'achat d'une enrubanneuse, ce n'est pas pareil. [...] là, [avec l'enrubannage], ça restait dehors. [...] C'était l'intérêt d'avoir de l'enrubannage ».

De nombreux éleveurs de bovins-viande l'utilisent pour cette raison. Dans le cas des éleveurs de vaches laitières, l'enrubannage permet aussi d'augmenter la qualité nutritionnelle du fourrage et d'intensifier la production:

«c'est vrai que les laitiers, ils en font tous [de l'enrubannage] parce que ça fait pisser du lait!».

Le développement de cette technique s'est accompagné de l'émergence de débats techniques et environnementaux parfois vifs, que ce soit entre éleveurs sur l'impact de l'enrubannage sur le prix des plastiques et la qualité de la viande et du lait, ou entre éleveurs et d'autres acteurs du territoire sur la pollution visuelle induite par exemple par ces tas de balles enrubannées qui jalonnent le paysage.

Le développement de cette technique a transformé de manière importante l'itinéraire technique traditionnel d'exploitation des prairies de fauche. Cette exploitation se traduisait par une première coupe fin juin-début juillet pour le foin, la repousse de l'herbe étant ensuite, soit coupée à nouveau (pour donner du regain ou « refoin »), soit pâturée à l'automne. L'enrubannage modifie tout d'abord les pratiques de fauche. Il permet en effet de couper le fourrage plus tôt et de réaliser parfois deux coupes supplémentaires. Il présente également l'intérêt de réduire le laps de temps entre la coupe et la récolte, et donc de réduire le risque d'exposition aux intempéries qui dégradent la qualité de l'herbe coupée. Il offre ainsi l'avantage d'améliorer la qualité nutritive du fourrage: d'une part, l'herbe peut être récoltée au meilleur stade dans la mesure où on s'affranchit (dans une certaine mesure) des aléas climatiques; d'autre part, à qualité d'herbe identique, l'enrubannage permet une conservation de la valeur nutritive du fourrage nettement supérieure à celle du foin (ce qui se traduit par une production de lait plus importante).

En ce qui concerne les pratiques de pâturage, l'enrubannage permet aux éleveurs laitiers, grâce à la fauche précoce, d'ouvrir plus rapidement (dès le début de l'été) au pâturage les prairies ainsi fauchées. Devant faire face à un manque de surfaces en pâturages au printemps, ces derniers effectuent à cette saison trois, voire quatre passages successifs sur les pâtures de printemps avant de pouvoir faire pâturer la repousse des prairies qui ont été fauchées pour produire l'enrubanné.

Sur le plateau du Vercors, des voies d'intensification fourragère font l'objet de controverses notamment par rapport à l'ancrage territorial des produits et à la biodiversité des prairies. Les partisans du développement des prairies temporaires, souvent associé à des pratiques de fertilisation par épandage de fumier ou lisier, d'enrubannage et de fauche précoce sont mis en cause par les défenseurs d'un maintien des prairies permanentes visant à valoriser la biodiversité de leurs prairies comme facteur de production ${ }^{13}$, voire comme facteur potentiel de différenciation des produits. 


\section{Deux cultures techniques de l'herbe}

La description des modalités sociotechniques d'usage et de valorisation des ressources herbagères nous amène à caractériser deux cultures techniques de l'herbe dans lesquelles les pratiques de pâturage prennent une place et un sens particuliers: d'une part, une « culture agropastorale » dans le Val d'Azun, qui repose essentiellement sur une gestion différenciée de l'espace et la mobilité des troupeaux, gestion qui s'appuie notamment sur le pâturage de la montagne; d'autre part, une "culture herbagère » dans les Quatre Montagnes, basée principalement sur une conduite différenciée des prairies dans laquelle le pâturage s'inscrit davantage dans une logique plus générale de production herbagère.

Le contexte du Val d'Azun est principalement marqué par des enjeux d'accès aux ressources. Comme nous l'avons vu, ce territoire est caractérisé par un partage des terres entre, d'un côté, des ressources fourragères cantonnées essentiellement aux estives et à des parcelles difficiles à travailler en zones intermédiaires et, d'un autre côté, des « bonnes terres » en fond de vallée où se pratique la culture du maïs. Dans ces conditions trois facteurs d'amélioration et de sécurisation de l'autonomie des exploitations sont mis en avant par les éleveurs: (1) l'amélioration des rendements des cultures ou l'acquisition de nouvelles parcelles cultivables; (2) la sécurisation de l'accès aux surfaces fourragères des zones intermédiaires soumises aux pressions foncières liées aux constructions de maisons et aux achats des granges et des parcelles par de nouveaux résidents; (3) l'allongement de la période de mise en estive des animaux et/ou la réouverture de certaines estives abandonnées pour multiplier les ressources fourragères associées. En dehors de l'usage des estives, la gestion de la ressource fourragère reste peu discutée entre les éleveurs.

La situation est très différente dans les Quatre Montagnes où c'est principalement l'intensification des formes d'exploitation et de valorisation des ressources herbagères qui sont en jeu. Cette situation se caractérise notamment par l'utilisation relativement confidentielle des estives collectives et de la culture des céréales. Contrairement au Val d'Azun, les débats relatifs à l'autonomie fourragère mettent en jeu des questions importantes de conduite des prairies: mélange d'espèces, implantation sous couvert de céréales, fertilisation, dates de fauche, modes de conservation, compromis fauche/pâture et quantité/qualité. La part importante de parcelles mécanisables ouvre le champ des possibles en matière de valorisation des ressources fourragères.

À travers ces mises en mouvement des logiques sociotechniques du travail et de l'usage de l'herbe, les identités professionnelles et territoriales des éleveurs du Vercors et de la Bigorre se retrouvent tantôt confortées, tantôt ébranlées. D’un côté, ces identités professionnelles et territoriales sont confortées lorsqu'elles renvoient à cette capacité technique des éleveurs à agencer la fonction productive de l'élevage à une diversité de fonctions non productives soutenues par les autres acteurs du territoire. Dans le Vercors, par exemple, les possibilités techniques de culture de l'herbe et le couplage des activités d'élevage et des activités touristiques, telles que les activités de sports d'hivers ou l'accueil des estivants (gîtes, visites et vente de fromages, etc.), ouvrent à une grande diversité de façons d'être éleveur. D'un autre côté, ces identités professionnelles et territoriales peuvent aussi être ébranlées et se recomposer quand des impératifs économiques ou sociaux poussent les éleveurs à « pratiquer les prairies » autrement qu'antérieurement. 
Dans une situation de concurrence de plus de plus marquée dans l'accès aux ressources fourragères nécessaires à la pérennité de l'activité, une lutte et une différenciation sociale entre éleveurs peut émerger dans l'accès aux terres cultivables ou aux meilleures estives. Mais des tensions entre certaines logiques d'exploitation agricole et pastorale de la montagne, d'une part, et certaines fonctions attendues de l'élevage portées par d'autres acteurs du territoire, d'autre part, peuvent également jouer. Dans le Val d'Azun, par exemple, le fait de garder une pression de pâturage suffisante dans les zones intermédiaires et dans les estives pour garantir le maintien de milieux ouverts (Barnaud $\&$ al., sous presse) est à l'origine de conflits importants entre éleveurs. Les partisans d'un effort d'entretien des zones intermédiaires s'opposent à ceux qui privilégient la sécurisation de leur système et la simplification du travail en allant par exemple chercher des parcelles en plaine.

Dans ces deux régions de montagne, la viabilité des exploitations et plus généralement les conditions de maintien des activités d'élevage dépendent de la capacité des éleveurs à réduire les charges liées à l'achat d'aliments. Les questions de l'autonomie fourragère des exploitations sont pour eux centrales et orientent la façon dont ils raisonnent leur activité et les modalités de leur inscription territoriale. Toutefois, au-delà de l'apparente ressemblance des contextes socio-écologiques des deux terrains étudiés, ce sont bien deux cultures techniques de l'herbe très différentes qui se révèlent, participant chacune d'une manière spécifique au maintien d'une activité d'élevage professionnelle en territoire de montagne. Lanalyse comparative permet ainsi de montrer que les éleveurs de la Bigorre et du Vercors n'abordent pas de la même façon l'articulation entre les lieux de production animale herbivore et les lieux où se trouvent les ressources herbagères et céréalières nécessaires à la bonne conduite de ces productions. À travers l'usage de ces ressources, ils ne sont pas confrontés aux mêmes acteurs et aux mêmes enjeux. Les logiques pratiques des éleveurs se trouvent ainsi, dans leur dynamique, étroitement liées à des logiques sociales qui animent leur façon de se penser éleveurs. Si les cultures techniques de l'herbe en région de montagne se caractérisent par un rapport particulier à l'autonomie fourragère, celle-ci se décline alors selon des modalités régionales différentes et renvoient à des formes d'autonomie et d'hétéronomie, à la fois techniques, identitaires et territoriales, contrastées.

Ce travail a été conduit dans le cadre du projet de recherche MOUVE financé par l'Agence Nationale de la Recherche (projet ANR-2010-STRA-005-01).

Auteurs: Antoine Doré (INRA), Claude Compagnone (AgroSup Dijon), Laurent Dobremez (Irstea centre de Grenoble), Sophie Madelrieux (Irstea centre de Grenoble), Cécile Barnaud(INRA). 


\section{NOTES}

Photo d'ouverture : Sur le plateau du Vercors, des voies d'intensification fourragère font l'objet de controverses notamment par rapport à l'ancrage territorial des produits et à la biodiversité des prairies.

1. L'ensemble de ces travaux ont été réalisés dans le cadre du projet de recherche MOUVE, soutenu financièrement par l'Agence Nationale de la Recherche (projet ANR-2010-STRA-005-01) et par la Zone-Atelier Alpes.

2. Un canton de 7 communes, $160 \mathrm{~km}^{2}, 1516$ habitants et 111 exploitations agricoles.

3. Un canton de 7 communes, $255 \mathrm{~km}^{2}, 11329$ habitants et 95 exploitations agricoles.

4. En 2010, la Surface Fourragère Principale (SFP) représente 98 \% de la Surface Agricole Utilisée et le maïsfourrage représente $1 \%$ de la SFP. L'ensemble des statistiques agricoles sur le Val d'Azun et les Quatre Montagnes mentionnées dans cet article sont issus des données Agreste des recensements agricoles de 1988, 2000 et 2010 et ont été traités par L. Dobremez et D. Borg (Irstea UR DTM Grenoble). Ce travail a été effectué en ayant recours au Centre d'accès sécurisé aux données (CASD) qui a bénéficié d'une aide de l'État gérée par l'Agence Nationale de la Recherche au titre du programme Investissements d'avenir portant la référence ANR-10-EQPX-17.

5. Seul un couple d'éleveurs pratique la traite et la fabrication des fromages en estive.

6. Sur la question du « faire propre » dans les mondes de l'élevage de montagne, voir notamment les travaux de Valérie Miéville-Ott (2000).

7. Les granges foraines sont les granges des zones intermédiaires qui composent (avec le bâtiment d'habitation principal et les cabanes d'estives) les biens immeubles de la « maison » pyrénéenne. Ces granges foraines permettent de stocker le foin des prairies de fauches attenantes et d'en limiter ainsi le transport dans ces zones peu praticables.

8. Entre 1988 et 2010, le nombre d'exploitation est passé de 190 à 95 (-50 \%), la taille moyenne du troupeau de vaches laitières est passée de 16 à 36 (+130\%), la Surface Agricole Utilisée (SAU) hors alpages collectifs est passée de 4748 ha à 4219 ha (-11\%).
9. Entre 1988 et 2010 , le rapport du nombre d'Unités de Travail Agricole (UTA) familiales sur le nombre total d'UTA passe de $97 \%$ à 93 \% et le nombre d'exploitations agricoles avec une activité exclusivement agricole passe de 92 à 25 (-73\%).

10. En 2010, la surface fourragère représente 96 \% de la surface agricole utilisée.

11. Entre 1988 et 2010, on est passé de $21 \%$ à $33 \%$ de prairies temporaires.

12. L'éleveur régule la hauteur d'herbe en ajustant la surface offerte et la complémentation en fourrage. Cette technique permet de simplifier le travail et d'offrir une herbe jeune favorable à la production de lait.

13. La diversité de la composition floristique des prairies est réputée les doter de propriétés agronomiques intéressantes comme une certaine résilience face aux aléas climatiques et une plus grande souplesse d'exploitation (la valeur nutritive du fourrage se maintenant plus longtemps au cours du cycle de végétation) (Pottier et al., 2012; Duru et al., 2010; Caldeira et al., 2005). 


\section{POUR CITER CET ARTICLE}

Doré, A., Compagnone, C., Dobremez, L. \&al. 2015 Pâturage et culture technique de l'herbe. In Dupré, L., Lasseur, J. \& R. Poccard-Chapuis, Pâturages Techniques \& Culture 63: 56-73

\section{RÉFÉRENCES}

Akrich, M. 1993 Les Formes de la médiation technique, Réseaux 60 : 87-98.

Barnaud C., Theil L., Choisis J.-P., \& al. (accepté) Les Services écosystémiques: une notion savante déconnectée des représentations locales? Une analyse des représentations locales de l'élevage en zone agricole défavorisée, In Dynamiques environnementales, politiques publiques, pratiques locales: quelles interactions? Paris: Quae.

Barthez, A. 1982 Famille, Travail et Agriculture. Paris: Economica.

Caldeira M.C., Hector A., Loreau M. \& al. 2005 Species richness, temporal variability and resistance of biomass production in a Mediterranean grassland, Oikos 110: 115-123.

Compagnone C. 2008 L'Analyse d'un système de pratiques sociales et techniques en élevage. Regard sociologique... sur une approche géographique, Nature Sciences Sociétés 16: 139-141.

Dobremez L. (coord.), Borg D., Madelrieux S., \& al.2012. Attentes des acteurs sur l'élevage dans le Parc naturel régional du Vercors: analyse des entretiens réalisés dans le cadre de la tâche 1 du projet ANR-Systerra-Mouve. Irstea DTM Grenoble, Isara-Lyon, Suaci Alpes du nord Saint-Baldoph, INRA SAD-APT VersaillesGrignon, avril 2012.

Dobremez L., Nettier B., Pauthenet Y., \& al. 2013. Organisation spatiale et fonctionnelle des exploitations agricoles dans le Val d'Autrans (Vercors). Projet ANR-Systerra-Mouve: rapport du terrain Vercors pour le livrable L4.2. Irstea UR DTM Grenoble, Suaci Alpes du nord Saint-Baldoph (73), octobre 2013.

Doré, A. 2013 Intensification écologique et redéfinition des identités professionnelles dans les mondes de l'élevage. Grenoble: Rapport Programme MOUVE (Les interactions Élevage et Territoire dans la mise en mouvement de l'intensification écologique - ANR SYSTERRA).

Duru M., Cruz P., Theau J.P. 2010 Évaluer la souplesse d'utilisation des prairies permanentes par la caractérisation de la composition fonctionnelle de la végétation et la phénologie des espèces, Fourrages $201: 3-10$.

Eychenne C., 2008 Les Éleveurs et l'estive: pour une approche compréhensive des pratiques pastorales, Natures Sciences Sociétés 16: 131 -138.

Fouilleux, E. 2008 Les Politiques agricoles et alimentaires, in O. Borraz \& V. Guiraudon (Dir.) Politiques publiques. 1, La France de la gouvernance européenne. Paris: Presses de Sciences Po: 113-146.

Gibon, A. 2005 Managing grassland for production, the environment and the landscape. Challenges at the farm and the landscape level, Livestock Production Science 96: 11- 31.

Hervieu, B. et Purseigle, F. 2013 Sociologie des mondes agricoles. Paris: Armand Colin.

Lémery, B. 2003 Les Agriculteurs dans la fabrique d'une nouvelle agriculture, Sociologie du Travail 45/1 : 9-25.

Miéville-Ott, V. 2000 Les Éleveurs du Jura face à l'écologisation de leur métier, Le Courrier de l'Environnement $40: 75-84$.

Mormont M. 2009 Globalisations et écologisations des campagnes, Études rurales 183/1 : 143-160.

Muller, P. 2010 Les Changements d'échelles des politiques agricoles - Introduction, in B. Hervieu, 
N. Mayer, P. Muller, F. Purseigle \& J. Rémy (Dir.) Les mondes agricoles en politique. Paris: Presses de Sciences-Po: 339-350.

Parguel P., Compagnone C. 2008 Un Dispositif d'appui aux agriculteurs pour l'élaboration de règles de production d'AOC, Économie Rurale 302: 10-22.

Pottier, E., Michaud A., Farrié J.P., et al. 2012 Les Prairies permanentes françaises au cour d'enjeux agricoles et environnementaux, Innovations Agronomiques 25: 85-97.

Rémy, J. 1987 La Crise de professionnalisation en agriculture: les enjeux de la lutte pour le contrôle du titre d'agriculteur, Sociologie du travail 4: 415-441.

Simondon, G. 1958 Du Mode d'existence des objets techniques. Paris: Aubier. 


\section{RÉSUMÉ}

Pâturages et cultures techniques de l'herbe. Les mondes de l'élevage de territoires de montagne du Vercors et de Bigorre. L'objectif de cet article est de décrire et d'analyser la façon dont les activités d'élevage herbivore des territoires de montagne s'appuient sur l'usage des ressources herbagères locales. En nous focalisant sur l'élevage de montagne, nous faisons apparaître la diversité des modalités pratiques d'articulation des lieux de production et des lieux de disponibilité des ressources dans des régions présentant des contrastes pédo-climatiques contraignants. Pour ce faire, nous rendons compte, dans une démarche comparative, des cultures techniques de l'herbe d'éleveurs du Vercors (Alpes du Nord) et d'éleveurs de la Bigorre (Pyrénées). La description des modalités sociotechniques d'usage et de valorisation des ressources herbagères nous amène à caractériser deux cultures techniques de l'herbe dans lesquelles les pratiques de pâturage prennent une place et un sens particuliers, étroitement liés à des logiques sociales qui animent leur façon de se penser éleveurs: une « culture agropastorale » dans le Val d'Azun, qui repose essentiellement sur une gestion différenciée de l'espace et la mobilité des troupeaux, et une « culture herbagère » dans les Quatre Montagnes, basée principalement sur une conduite différenciée des prairies.

\section{ABSTRACT}

Grazing and technical grass cultures. The world of livestock breeding in the mountain regions of the Vercors and the Bigorre. The aim of this article is to describe and analyze the way in which livestock grazing activities in mountain regions depend on the use of local grassland resources. By focusing on mountain livestock farming, we attempt to show the diversity of practical means of linking production sites to sites where resources are available in regions with major environmental constraints. To do this, we used a comparative approach to describe the technical grass cultures of livestock farmers in the Vercors (Northern Alps, France) and in the Bigorre (Pyrenees, France). The description of the sociotechnical means for using and developing grassland resources led us to characterize two technical grass cultures in which grazing practices have a particular role and meaning, closely linked to the social rationales that motivate the way livestock farmers see themselves: an « agropastoral culture » in the Val d'Azun, which essentially relies on the differentiated management of space and of herd mobility, and a " grass culture » in the Quatre Montagnes, primarily based on differentiated grassland management.

\section{MOTS-CLÉS}

culture technique, identité professionnelle, éleveurs de bétail, ressources herbagères, élevage de bétail de montagne

\section{KEYWORDS}

technical cultur, professional identity, livestock farmers, grassland resources, mountain livestock farming 